\title{
RELAÇÃO ENTRE DENSIDADE DO SOLO E CONTEÚDO DE ÁGUA EM REPETIDOS CICLOS DE CONTRAÇÃO E EXPANSÃO EM UM LATOSSOLO
}

\author{
Paulo Ivonir Gubiani(1)*, Quirijn de Jong Van Lier(2), Marta Sandra Drescher(3), Henrique \\ Caletti Mezzomo(1) e Camila Machado Costa Veiga(1)
}

\footnotetext{
(1) Universidade Federal de Santa Maria, Departamento de Solos, Campus Universitário, Santa Maria, Rio Grande do Sul, Brasil. (2) Universidade de São Paulo, Centro de Energia Nuclear na Agricultura, Laboratório de Física do Solo, Piracicaba, São Paulo, Brasil.

(3) Universidade Estadual do Rio Grande do Sul, Campus Regional IV, Unidade de São Luiz Gonzaga, Porto Alegre, Rio Grande do Sul, Brasil.

* Corresponding author.

E-mail: paulogubiani@gmail.com
}

\section{RESUMO}

A compactação do solo é uma consequência indesejável do uso agrícola do solo, sobretudo em sistemas de cultivo com mínimo revolvimento do solo, como é o caso do plantio direto (PD). Contudo, a compactação que o tráfego de máquinas causa no solo sob PD não inviabiliza a produção das culturas, indicando que mecanismos intrínsecos a ele promovem reversão da compactação. Neste estudo, avaliou-se a influência de ciclos de contração e expansão sobre a densidade do solo ( $\rho)$ de um Latossolo Vermelho argiloso $\left(0,57 \mathrm{~kg} \mathrm{~kg}^{-1} \mathrm{de}\right.$ argila e $0,12 \mathrm{~kg} \mathrm{~kg}^{-1}$ de areia) e a mudança temporal da $\rho$ no campo. Amostras de solo foram compactadas no laboratório até atingirem $\rho$ de $1464 \mathrm{~kg} \mathrm{~m}^{-3}$ e submetidas a cinco ciclos de contração (secagem ao ar) e expansão (saturação). Durante a contração, foi monitorado o conteúdo gravimétrico de água e a $\rho$. A $\rho$ foi medida também no campo, nos anos de 2010, 2011 e 2013. O decréscimo do conteúdo de água nas amostras provocou aumento da $\rho$ conforme uma função sigmoide com duas assíntotas, e o aumento da $\rho$ foi expressivo em conteúdos de água menores que o do ponto de murcha permanente (1,5 MPa). Embora tenha havido aumento da $\rho$ durante a contração, os sucessivos eventos de contração e expansão reduziram gradativamente a $\rho$ de 1713 para $1570 \mathrm{~kg} \mathrm{~m}^{-3}$ (final da contração), e de 1464 para $1385 \mathrm{~kg} \mathrm{~m}^{-3}$ (próximo à saturação). Em solo compactado no campo também foi verificado a variação decrescente de $\rho$ (de 1406 para $1327 \mathrm{~kg} \mathrm{~m}^{-3}$ ) a uma taxa de $-26 \mathrm{~kg} \mathrm{~m}^{-3}$ ano $^{-1}$. Concluiu-se que a diminuição do grau de compactação no campo está ligada em grande parte ao mesmo mecanismo que diminuiu o grau de compactação das amostras no laboratório. Assim, no 


\begin{abstract}
solo usado neste estudo, a contração e expansão conseguiram reverter grande parte da compactação que o tráfego de máquinas causa nele.
\end{abstract}

Palavras-chave: compactação do solo, resiliência, plantio direto.

\title{
ABSTRACT: RELATIONSHIP BETWEEN BULK DENSITY AND WATER CONTENT OF AN OXISOL SUBJECTED TO REPEATED CYCLES OF EXPANSION AND CONTRACTION
}

\begin{abstract}
Soil compaction is an undesirable consequence of land use for crop production, especially under cultivation with minimum soil turnover as in the no-tillage system (NTS). However, the compaction caused by machine traffic on soils under NTS does not make crop production unviable, indicating that some intrinsic mechanisms promote the reversion of soil compaction. In this study, the influence of alternate contraction and expansion cycles was evaluated with regard to the bulk density ( $\rho$ ) of a Latossolo Vermelho argiloso (clayey Oxisol) (0.57 kg kg-1 clay, and $0.12 \mathrm{~kg} \mathrm{~kg}^{-1}$ sand) and the temporal change in $\rho$ in the field. Soil samples were compacted in the laboratory to reach a $\rho$ of $1,464 \mathrm{~kg} \mathrm{~m}^{-3}$ and subjected to five cycles of contraction (air drying) and expansion (saturation). During the contraction process, soil gravimetric water content and $\rho$ were monitored. Soil $\rho$ was also measured in the field in the years 2010, 2011, and 2013. A decrease in soil water content in the samples increased the $\rho$ according to a sigmoid function with two asymptotes, and the increase in $\rho$ was significant at water contents lower than that of the permanent wilting point (1.5 MPa). Although there was an increase in $\rho$ during contraction, successive contraction and expansion events steadily reduced $\rho$ from 1,713 to $1,570 \mathrm{~kg} \mathrm{~m}^{-3}$ (end of contraction), and from 1,464 to $1,385 \mathrm{~kg} \mathrm{~m}^{-3}$ (near saturation). In the field, a decline in $\rho$ was observed in compacted soil (from 1,406 to $1,327 \mathrm{~kg} \mathrm{~m}^{-3}$ ) at a rate of $-26 \mathrm{~kg} \mathrm{~m}^{-3} \mathrm{yr}^{-1}$. We conclude that the decrease in the state of field compaction is largely linked to the same mechanisms that cause the decrease in compaction in the samples in the laboratory. We conclude that for the soil used in this study, contraction and expansion cycles may reverse most of the compaction that machine traffic causes to the soil.
\end{abstract}

Keywords: soil compaction, resiliency, no-tillage.

\section{INTRODUÇÃO}

Ao longo dos últimos 40 anos, houve diminuição expressiva do revolvimento do solo em áreas agrícolas em razão da adoção do plantio direto (PD), que ocupa aproximadamente 30 milhões de hectares no Brasil (Febrapdp, 2014) e representa 80 \% da área cultivada com soja, milho e trigo na região Sul do país (Cooplantio, 2012). As características de manejo do solo no PD, como a pequena intervenção mecânica, basicamente restrita à ação dos dispositivos de posicionamento de sementes e fertilizantes, e os sucessivos tráfegos de implementos agrícolas (semeadura, aplicação de fertilizantes e defensivos, colheita e transporte) são indicativos de que a compactação no PD deve ser um problema maior do que foi no sistema convencional de cultivo.

Em avaliações em campo, detecta-se em muitas áreas de PD a presença de uma camada mais densa localizada entre as profundidades de 0,08 e $0,15 \mathrm{~m}$, em comparação à camada superficial (0-0,05 m), que tem menor densidade e elevada porosidade total (Genro Junior et al., 2004; Suzuki et al., 2008; Secco et al., 2009). Nessas áreas, estudos têm indicado que a presença de camadas compactadas logo abaixo da superfície pode restringir o crescimento radicular das plantas (Reichert et al., 2007) e até mesmo confinar as raízes em pequeno volume de solo superficial e causar redução do crescimento da parte aérea e do rendimento das culturas (Klein et al., 2009).

Em razão da maior permanência da compactação em PD, visto que o solo é minimamente revolvido, estudos têm sugerido indicadores de compactação crítica para o crescimento das plantas (Silva et al., 1994; Reichert et al., 2009). Existem, contudo, inúmeras evidências em lavouras comerciais sob PD de que a compactação não atinge os níveis considerados críticos pela pesquisa (Gubiani et al., 2013a). Essas evidências sugerem que o tráfego nas lavouras comerciais não causa compactação no solo comparável à que ocorre nos experimentos onde são obtidos os indicadores, ou que o solo consegue se recuperar da degradação estrutural causada pelo tráfego.

Um possível mecanismo de recuperação da estrutura do solo é a contração e expansão, que têm sido avaliadas, principalmente em experimentos de laboratório. Alterações na estrutura do solo são relatadas desde a escala de microestrutura, observada com microscopia ótica (Viana et al., 2004), tomografia computadorizada (Pires e Bacchi, 2010) 
até a escala de macroagregados (Bastos et al., 2005). Outros estudos afirmam que repetidos ciclos de contração e expansão promovem a recuperação estrutural do solo (Chinn e Pillai, 2008) e que a intensidade da contração e expansão parece independer do estado de compactação do solo (Peng et al., 2006). Isso é de grande importância prática, pois em áreas compactadas em $\mathrm{PD}$, a formação de fraturas resultantes da contração e expansão pode aumentar a capacidade de infiltração do solo (Gebhardt et al., 2012) e criar caminhos de menor resistência ao crescimento de raízes (Bengough et al., 2011). Consequentemente, o estado de compactação percebido pelas plantas é menor que o de compactação global avaliado com o uso de indicadores físicos do solo.

Acredita-se que a capacidade de recuperação do solo após a compactação (resiliência) seja mais intensa em solos em que há quantidades expressivas de argilominerais expansivos, como os Vertissolos, Luvissolos e Cambissolos (Kämpf e Curi, 2003). Contudo, solos com elevado teor de caulinita, como os Latossolos, também manifestam contração e expansão, por causa da variação das forças associadas aos meniscos de água nas interfaces sólido-líquido-ar e ao arranjo flexível de cristalitos de caulinita de pequena dimensão (Kämpf e Curi, 2003). Investigando a sensibilidade de indicadores usados para avaliar a capacidade de recuperação de dois Latossolos sob PD (porosidades total e de aeração, conteúdo volumétrico de água, teor de carbono orgânico, permeabilidade ao ar e densidade do solo), Bavoso et al. (2012) também verificaram que a recuperação da estrutura do solo promovida por ciclos de umedecimento e secagem foi mais acentuada no Latossolo com maior teor de argila. Assim, na grande extensão de Latossolos manejados sob PD, principalmente nos argilosos, pode haver benefício expressivo da contração e expansão para a descompactação do solo. Diante disso, um dos grandes interesses é saber se esse processo natural tem potencial para compensar a compactação causada pelo tráfego e dispensar o consumo de tempo e energia com operações mecanizadas de descompactação, como a escarificação.

Portanto, se há evidências de que a estrutura do solo em áreas manejadas sob PD sofre degradação, mas não segue um caminho irreversível de compactação e redução na produção das culturas, é necessário estudar e compreender a participação de processos naturais da recuperação da estrutura do solo, bem como as possibilidades de manejar esses processos em escala de lavoura. Nesse contexto, o objetivo deste trabalho foi avaliar a influência de sucessivos ciclos de contração e expansão sobre a densidade de um Latossolo argiloso em experimento de laboratório e compará-la com a modificação temporal da densidade do solo no campo.

\section{MATERIAL E MÉTODOS}

O estudo foi desenvolvido em um Latossolo Vermelho distroférrico típico $\left(0,12 \mathrm{~kg} \mathrm{~kg}^{-1} \mathrm{de}\right.$ areia, $0,31 \mathrm{~kg} \mathrm{~kg}^{-1}$ de silte e $0,57 \mathrm{~kg} \mathrm{~kg}^{-1}$ de argila), cultivado em plantio direto (PD), situado em Ijuí, RS

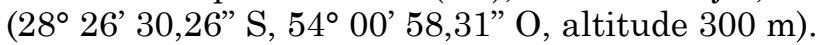
Nesse local havia sido implantado um experimento com diferentes graus de compactação, constituídos pela condição atual da área sob plantio direto, plantio direto seguido de escarificação e plantio direto com aplicação de compactação adicional por tráfego, onde foi estabelecida a cultura de milho nas safras 2010/2011 e 2011/2012. A escarificação foi efetuada somente na safra 2010/2011, até a profundidade média de $0,30 \mathrm{~m}$. A compactação adicional foi efetuada com três passadas (paralelas, deslocadas uma da outra por meia largura do pneu) de um trator MF292 na safra 2010/2011 e foi repetida (uma passada de tráfego do mesmo trator), na safra 2011/2012. Mais detalhes do experimento podem ser obtidos em Gubiani (2012).

Em cada safra, foram coletadas 96 amostras de solo com estrutura preservada (anéis metálicos de $0,06 \mathrm{~m}$ de diâmetro e $0,05 \mathrm{~m}$ de altura), nas profundidades de $0,07 \mathrm{~m}$ (48 amostras) e $0,25 \mathrm{~m}$ (48 amostras), definidas tendo-se como referência a distância a partir da superfície do solo até a posição central da altura do anel. No total, foram coletadas 192 amostras de solo nas duas safras. Após o experimento, a área permaneceu sem revolvimento do solo e foi cultivada com a cultura de soja na safra 2012/2013, permanecendo em pousio no outono de 2013. Em setembro de 2013, outras 36 amostras (18 a $0,07 \mathrm{~m}$ e 18 a $0,25 \mathrm{~m}$ ) foram coletadas. O número de repetições em cada tratamento foi 16 , 16 e 6, respectivamente, na primeira, segunda e terceira coletas. Nessas amostras, foi determinada a densidade do solo $(\rho)$, dividindo-se a massa do solo seco em estufa a $105^{\circ} \mathrm{C}$ pelo volume interno do anel.

Aproximadamente $20 \mathrm{~kg}$ de solo foram coletados, em setembro de 2013, em um único ponto do experimento, na camada de 0,0-0,2 m. Essa coleta única se justifica visto que o solo em toda a área era homogêneo quanto à granulometria e ao teor de matéria orgânica. Com essa quantidade de solo, foi conduzido um experimento no laboratório para avaliar o efeito de ciclos de contração e expansão na densidade do solo ( $\rho$ ). O solo foi seco ao ar e passado em peneira de malha de $2 \mathrm{~mm}$ (TFSA). A TFSA foi umedecida e homogeneizada até atingir o conteúdo de água $\left(U, \mathrm{~kg} \mathrm{~kg}^{-1}\right)$ ótimo para máxima compactação, estimado em razão do teor de argila $\left(A, \mathrm{~kg} \mathrm{~kg}^{-1}\right)$ por meio da equação $U=0,0358+0,4167 A$, descrita por Klein (2014). Em cinco anéis metálicos de 0,076 m de diâmetro por 0,076 m de altura (cinco repetições de solo), a TFSA umedecida foi compactada com auxílio de uma prensa mecânica ("morsa"). A quantidade de solo adicionada em cada anel foi calculada para que, 
após preenchimento, a densidade fosse de $1464 \mathrm{~kg} \mathrm{~m}^{-3}$, que corresponde a $98 \%$ da densidade de máxima compactação desse solo $\left(\rho_{m}, \mathrm{~kg} \mathrm{~m}^{-3}\right)$. A $\rho_{m}\left(1490 \mathrm{~kg} \mathrm{~m}^{-3}\right)$ foi estimada em razão do teor de argila por meio da equação $\rho_{m}=2.013,8$ - $920 \mathrm{~A}$, descrita por Klein (2014). A massa total de solo de cada anel foi dividida em duas partes para que a compactação fosse feita em duas etapas (camadas com espessura de $0,038 \mathrm{~m}$ ), com intuito de homogeneizar a compactação na amostra.

Depois de compactadas, as amostras foram saturadas por capilaridade por $24 \mathrm{~h}$ e, posteriormente, mantidas sobre uma grade metálica para evaporação gradativa da água em ambiente de laboratório. Durante esse processo de evaporação, a altura das amostras foi medida diariamente com um paquímetro em dois pontos fixos na superfície do solo. Quando a altura das amostras não variava por dois dias consecutivos, encerrava-se essa fase, tomando-se também a medida da contração do diâmetro da superfície do cilindro de solo dentro do anel. Na sequência, as amostras eram saturadas e iniciava novo ciclo de expansão e posterior contração. Ao total, foram aplicados cinco ciclos de expansão e contração (cinco repetições de contração e expansão), com os quais foram obtidas 25 curvas de densidade em função do conteúdo de água.

O conteúdo gravimétrico de água nas amostras $U\left(\mathrm{~kg} \mathrm{~kg}^{-1}\right)$ foi determinado diariamente pela pesagem das amostras, e a densidade $\rho\left(\mathrm{kg} \mathrm{m}^{-3}\right)$, na mesma frequência, dividindo-se a massa do solo seco em estufa a $105^{\circ} \mathrm{C}$ (obtida no final do experimento) pelo volume respectivo da amostra. $\mathrm{O}$ volume da amostra foi calculado descontando-se do volume interno total do anel o volume não ocupado pelo solo em razão da diminuição de diâmetro e da altura. Por questões operacionais, o diâmetro foi medido na superfície do solo apenas no final de cada ciclo de contração, obtendo-se o afastamento da amostra da parede interna do anel $(\Delta D, \mathrm{~m})$. Também no final de cada ciclo de contração obteve-se a redução da altura da amostra $(\Delta h, \mathrm{~m})$. Presumiu-se que o diâmetro da amostra no dia $i\left(D_{i}, \mathrm{~m}\right)$ foi reduzido proporcionalmente com o decréscimo diário da altura $\left(h_{i}, \mathrm{~m}\right)$, ou seja, $D_{i}=D_{c}-(\Delta D / \Delta h) h_{\mathrm{i}}$, em que $D_{c}(\mathrm{~m})$ é o diâmetro interno do anel.

A partir da análise preliminar dos dados, verificou-se que o formato da curva da densidade $\rho$ em função ao conteúdo de água $U$ assemelha-se à função sigmoide, apresentando um ponto de inflexão e duas assíntotas horizontais; a superior correspondente à densidade final quando a umidade tende a zero e a inferior correspondente à densidade inicial com a umidade tendendo à saturação (Figura 1).

Esse tipo de curva tem três pontos característicos. No sentido de decréscimo de $U$, inicialmente tem-se uma das raízes da terceira derivada da função $\rho(U)$, denominado de primeiro ponto de máxima curvatura (PMC1). Esse ponto representa o valor de $U$ a partir do qual há aumento máximo local na taxa de variação de $\rho$ em relação a $U$. Na sequência, tem-se o ponto de inflexão $(P I)$, que é a raiz da segunda derivada da função $\rho(U)$. Esse ponto representa o valor de $U$, em que a taxa de variação da $\rho$ é máxima. Por fim, tem-se a outra raiz da terceira derivada da função $\rho(U)$, denominado de segundo ponto de máxima curvatura $(P M C 2)$. Esse ponto representa o valor de $U$ a partir do qual há decréscimo máximo local na taxa de variação da $\rho$, indicando que a contração se aproxima do seu limite de contração do solo.

No intuito de se estabelecer uma forma objetiva para a estimativa dos valores de PI, PMC1 e PMC2, procurou-se ajustar uma equação matemática adequada, cujas segunda e terceira derivadas possuíssem raízes que pudessem ser determinadas analiticamente. Uma equação apropriada é a função sigmoide:

$$
\rho=\rho+\left(\rho_{f}-\rho_{i}\right)\left[1 / 2 \frac{\arctan \{a(d-U)\}}{\pi}\right]
$$

em que $\rho_{i}\left(\mathrm{~kg} \mathrm{~m}^{-3}\right)$ e $\rho_{f}\left(\mathrm{~kg} \mathrm{~m}^{-3}\right)$ são os valores das assíntotas inferior e superior, respectivamente; e $a\left(\mathrm{~kg} \mathrm{~kg}^{-1}\right)$ e $d\left(\mathrm{~kg} \mathrm{~kg}^{-1}\right)$, parâmetros de ajuste da equação. Demonstra-se que a raiz da segunda derivada dessa equação é dada pelo próprio valor de $d$, ou seja:

$$
P I=d
$$

e os valores das raízes da terceira derivada, $P M C 1$ e PMC2, são iguais a

$$
\begin{aligned}
& P M C 1=d+\frac{\sqrt{3}}{3 a} \\
& P M C 2=d+\frac{\sqrt{3}}{3 a}
\end{aligned}
$$

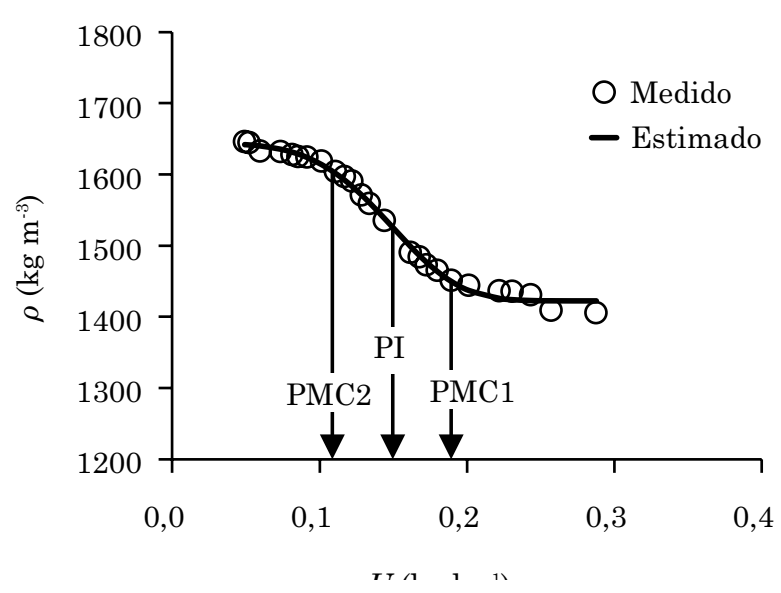

Figura 1. Pontos observados de densidade do solo $(\rho)$ versus conteúdo gravimétrico de água $(U)$, com indicação aproximada dos pontos de máxima curvatura (PMC1 e PMC2) e do ponto de inflexão (PI). 
Observe que a equação 1 tem simetria horizontal a partir de $U=d$, conforme verificado nas equações $3 \mathrm{e}$ 4. A equação 1 foi ajustada aos dados experimentais, utilizando-se o método Gaus-Newton do software SAS (SAS, 1999). A fim de não impor restrições ao posicionamento da curva, $\rho_{i}$ e $\rho_{f}$ também foram estimados como parâmetros de ajuste, do mesmo modo que foram estimados $a$ e $d$.

Para verificar o potencial matricial no $P I$, PMC1 e PMC2, uma porção do mesmo solo usado para preparar as amostras dos ciclos de contração e expansão foi utilizada para obter a relação do conteúdo gravimétrico com o potencial da água no solo, em potenciais menores (mais negativos) que -0,5 MPa. Para isso, o potencial da água de amostras de solo preparadas com diferentes conteúdos de água foi medido em um psicrômetro (Water Potential Meter - WP4), conforme descrito em Decagon (2000).

\section{RESULTADOS E DISCUSSÃO}

Em todas as amostras, observou-se aumento da densidade $(\rho)$ com o decréscimo no conteúdo de água. Desde a saturação até os menores conteúdos de água em que foram feitas as medições, o aumento médio na $\rho$ foi de 250; 200; 230; 200; e $180 \mathrm{~kg} \mathrm{~m}^{-3}$, na sequência do primeiro para o quinto evento de contração (Figura 2). Embora a $\rho$ aumentasse durante a contração, os sucessivos eventos de contração e expansão reduziram gradativamente a $\rho$ de 1713 para $1570 \mathrm{~kg} \mathrm{~m}^{-3}$ (valores das medições finais da contração), e de 1464 para $1385 \mathrm{~kg} \mathrm{~m}^{-3}$ (valores na condição de solo próximo à saturação) (Figura 3). Assim, a sucessão de contração e expansão parece ter sido eficiente para diminuir o nível de compactação do solo.

Com base na densidade de partículas $\left(\rho_{p}\right)$ de $2700 \mathrm{~kg} \mathrm{~m}^{-3}$, determinada por Gubiani (2012), e nas
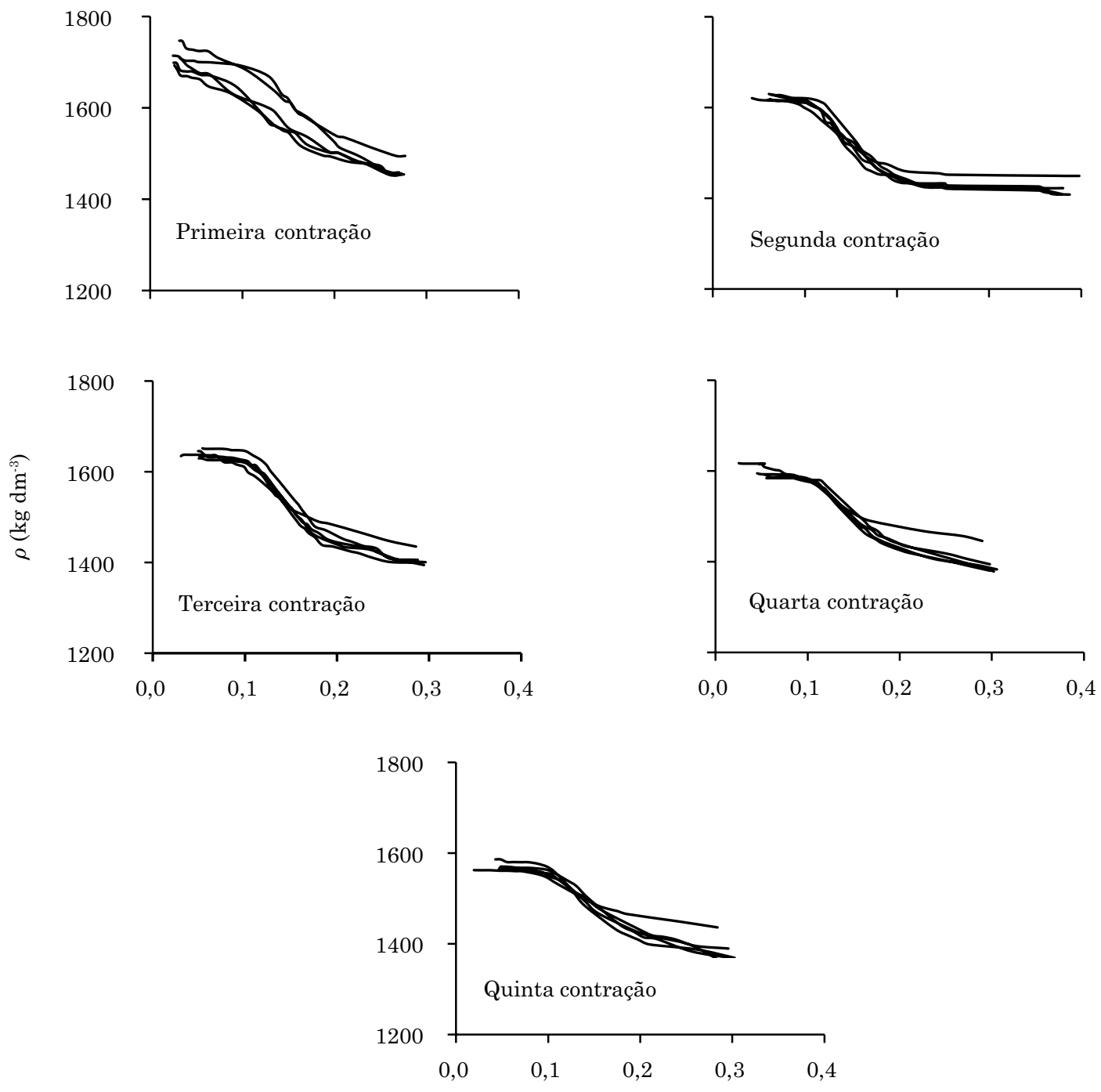

Figura 2. Relação da densidade do solo ( $(\rho)$ com o conteúdo gravimétrico de água (U) nos cinco eventos de contração. Cada curva representa um cilindro de solo. 


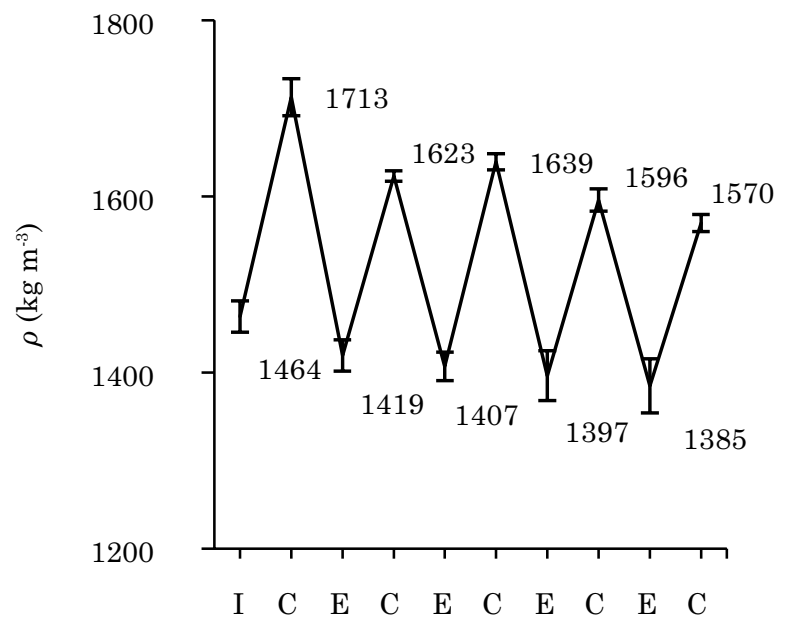

Figura 3. Densidade do solo inicial das amostras (I) e ao final dos sucessivos eventos de contração (C) e de expansão (E). Barras verticais representam o desvio-padrão.

médias de $\rho$ mencionadas anteriormente, a porosidade total do solo $\left(1-\rho / \rho_{p}\right)$ aumentou de 0,37 para $0,42 \mathrm{~m}^{3} \mathrm{~m}^{-3}$ (no fim da contração) e de 0,46 para $0,49 \mathrm{~m}^{3} \mathrm{~m}^{-3}$ (próximo à saturação). Esses resultados indicam que as forças matriciais, associadas à curvatura dos meniscos de água nas interfaces sólido-líquido-ar no solo, podem promover mudança significativa no volume do solo que, ocorrendo repetitivamente, pode se constituir num mecanismo eficiente de descompactação natural de Latossolos como o usado neste estudo.

Com os valores estimados dos parâmetros de ajuste da equação 1 (Quadro 1), foram calculados o $P I$ com a equação 2 , o $P M C 1$ com a equação 3 e o PMC2 com a equação 4. Exceto para o primeiro ciclo de contração, os valores de conteúdo de água $U$ correspondentes ao PMC1, PMC2 e PI não se alteraram muito (Figura 4a). O valor médio, seguido pelo desvio-padrão, do $P M C 1$ foi $0,170( \pm 0,012) \mathrm{kg} \mathrm{kg}^{-1}$; do PI, 0,144 $( \pm 0,013) \mathrm{kg} \mathrm{kg}^{-1}$; e do PMC2, 0,118 $( \pm 0,023) \mathrm{kg} \mathrm{kg}^{-1}$. Todos eles foram menores que o conteúdo de água no potencial matricial de -1,5 $\mathrm{MPa}\left(0,183 \mathrm{~kg} \mathrm{~kg}^{-1}\right)$, estimado com a equação ajustada ao conjunto de dados de conteúdo de água e potencial matricial obtido com o WP4 (Figura 4b). A contração do solo com a redução do conteúdo de água até $0,18 \mathrm{~kg} \mathrm{~kg} \cdot{ }^{-1}$ causou aumento de $12 \%$ na $\rho$.

O aumento da $\rho$ com o decréscimo do conteúdo de água está relacionado com a aproximação de partículas e o aumento da coesão do solo, com consequente aumento da resistência mecânica do solo (Horn e Smucker, 2005). Se isso ocorre de forma expressiva na faixa de conteúdo de água de crescimento das plantas, então o poder preditivo dos modelos de estimativa da resistência à penetração ao crescimento de raízes aumentaria

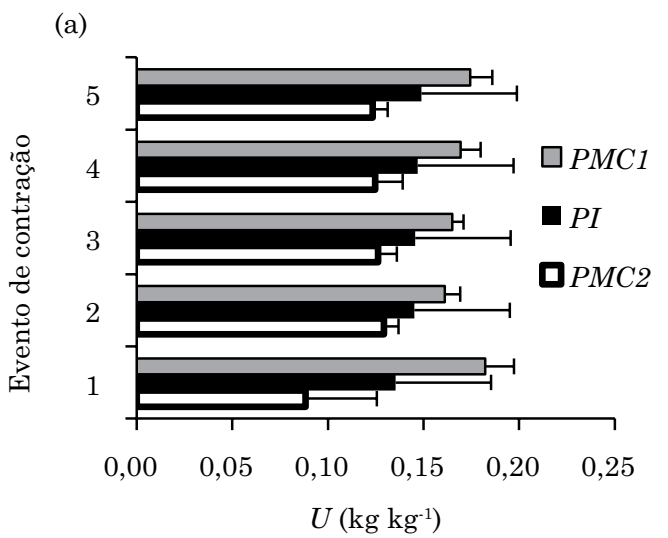

(b)

$$
U\left(\mathrm{~kg} \mathrm{~kg}^{-1}\right)
$$

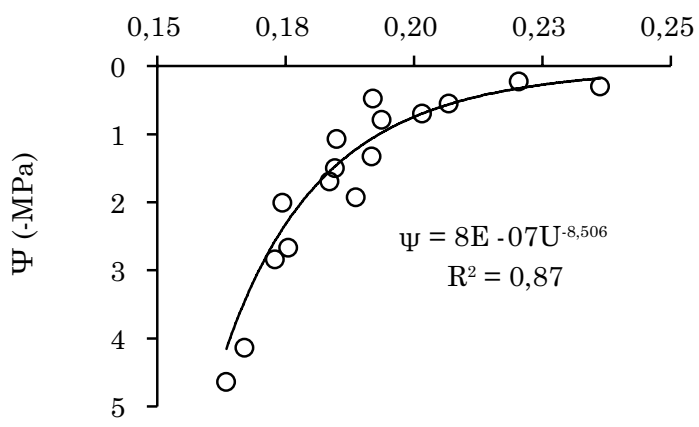

Figura 4. Conteúdo gravimétrico de água no solo $(U)$ no primeiro (PMC1) e no segundo (PMC2) ponto de máxima curvatura e no ponto de inflexão $(P I)$, obtidos por ajuste da equação (1) aos dados experimentais (a); e relação entre $U$ e o potencial matricial $(\Psi)(b)$. Linhas no extremo direito das barras representam o desvio-padrão.

pela incorporação desses processos. Para subsidiar essa incorporação, a necessidade de usar o valor de $\rho$ para a condição de conteúdo de água em que a resistência à penetração é medida deveria ser investigada. Usualmente, a resistência à penetração é medida em amostras com diferentes conteúdos de água, mas a $\rho$ dessas amostras é medida apenas na condição de solo seco a $105^{\circ} \mathrm{C}$. $\mathrm{O}$ ajuste de um modelo como o de Busscher (1990) sem se considerar que $\rho$ é função do conteúdo de água pode resultar numa equação de estimativa da resistência à penetração que atribui ao conteúdo de água uma parcela de efeito que de fato deveria ser atribuída à $\rho$. Esse problema assume maior importância se as estimativas da resistência à penetração em função do conteúdo de água e da densidade do solo forem estendidas para conteúdos de água menores que o do PMC1.

No campo, o processo de descompactação por ação de ciclos de contração e expansão pode ser demorado, pois a variação do conteúdo de água no solo deve ser grande para haver recuperação da 
estrutura, desde o PMC1 até PMC2. Disso decorre que a resiliência promovida pela contração e expansão é mais eficiente nas camadas próximas da superfície do solo, onde ocorrem maiores variações do conteúdo de água ao longo do tempo. Em profundidade, a recuperação da estrutura do solo pode acontecer apenas em períodos prolongados sem chuva, como nas estiagens e secas, que promovem variação expressiva do conteúdo de água. Diferentemente, nas áreas de PD, nas quais a presença de camadas compactadas é verificada logo abaixo da superfície (entre 0,08 e $0,15 \mathrm{~m}$ ) (Genro Junior et al., 2004; Suzuki et al., 2008; Secco et al., 2009), é de se esperar que os processos de contração e expansão estejam revertendo parte da compactação dos solos.

Isso pode explicar, em parte, o fato de a compactação não atingir níveis críticos em muitas áreas comerciais ou experimentais sob PD, utilizadas com ou sem integração lavoura-pecuária (Gubiani et al., 2013a). Um caso que corrobora com essa suposição foi observado no experimento em campo com o Latossolo Vermelho distroférrico típico, em que a $\rho$ foi monitorada em três anos. Tanto na safra 2010/2011 como na safra 2011/2012 não houve precipitação no período posterior à compactação e ao plantio da cultura do milho (Gubiani et al., 2013b), o que pode ter provocado a ocorrência de contração e expansão. Na profundidade de 0,07 m, de 2010 para 2013, a densidade média do solo compactado (PDC) decresceu de $1406 \mathrm{~kg} \mathrm{~m}^{-3}$ para $1327 \mathrm{~kg} \mathrm{~m}^{-3}$, se aproximando do valor de $1290 \mathrm{~kg} \mathrm{~m}^{-3}$ observado no plantio direto, que não recebeu compactação adicional (PD) (Figura 5). Em valores absolutos médios, a taxa de variação da densidade no PDC $\left(-26 \mathrm{~kg} \mathrm{~m}^{-3} \mathrm{ano}^{-1}\right)$ em termos absolutos se aproximou da taxa de variação da densidade no PDE $\left(+31 \mathrm{~kg} \mathrm{~m}^{-3}\right.$ ano $\left.^{-1}\right)$. Isso sugeriu que os mecanismos que levaram à diminuição da densidade verificada no experimento de laboratório também atuam no campo, possibilitando a recuperação da estrutura do solo.

Em solos diferentes do estudado neste trabalho, principalmente naqueles que contêm baixo teor de argila, a reversão da compactação e recuperação da estrutura do solo pode ser pequena (Bavoso et al., 2012). Nesses solos, as estratégias de descompactação e a frequência com que elas devem ser aplicadas são maiores em relação a solos mais resilientes. Para o Latossolo Vermelho distroférrico típico deste estudo, a diminuição da densidade no campo indicou que, naturalmente, o solo seguiu um curso de recuperação, sem requerer técnicas de descompactação (Figura 5). A recuperação verificada,

Quadro 1. Valores estimados dos parâmetros de ajuste da equação 1 para todas as amostras e ciclos de contração e expansão

\begin{tabular}{|c|c|c|c|c|c|c|}
\hline \multirow[t]{2}{*}{ Ciclo } & \multirow[t]{2}{*}{ Parâmetro } & \multicolumn{5}{|c|}{ Cilindro de solo } \\
\hline & & 1 & 2 & 3 & 4 & 5 \\
\hline \multirow[t]{4}{*}{1} & $\rho_{i}\left(\mathrm{~kg} \mathrm{~m}^{-3}\right)$ & 1331 & 1400 & 1406 & 1435 & 1408 \\
\hline & $\rho_{f}\left(\mathrm{~kg} \mathrm{~m}^{-3}\right)$ & 1881 & 1774 & 1755 & 1810 & 1747 \\
\hline & $a\left(\mathrm{~kg} \mathrm{~kg}^{-1}\right)$ & 7,595 & 12,90 & 13,41 & 13,73 & 19,82 \\
\hline & $d\left(\mathrm{~kg} \mathrm{~kg}^{-1}\right)$ & 0,1162 & 0,1248 & 0,1220 & 0,1423 & 0,1715 \\
\hline \multirow[t]{4}{*}{2} & $\rho_{i}\left(\mathrm{~kg} \mathrm{~m}^{-3}\right)$ & 1411 & 1412 & 1401 & 1440 & 1402 \\
\hline & $\rho_{f}\left(\mathrm{~kg} \mathrm{~m}^{-3}\right)$ & 1656 & 1644 & 1647 & 1643 & 1657 \\
\hline & $a\left(\mathrm{~kg} \mathrm{~kg}^{-1}\right)$ & 36,25 & 42,05 & 34,66 & 33,25 & 35,36 \\
\hline & $d\left(\mathrm{~kg} \mathrm{~kg}^{-1}\right)$ & 0,1434 & 0,1408 & 0,1507 & 0,1359 & 0,1551 \\
\hline \multirow[t]{4}{*}{3} & $\rho_{i}\left(\mathrm{~kg} \mathrm{~m}^{-3}\right)$ & 1392 & 1386 & 1392 & 1425 & 1386 \\
\hline & $\rho_{f}\left(\mathrm{~kg} \mathrm{~m}^{-3}\right)$ & 1675 & 1660 & 1666 & 1675 & 1691 \\
\hline & $a\left(\mathrm{~kg} \mathrm{~kg}^{-1}\right)$ & 28,40 & 35,42 & 34,95 & 23,68 & 29,54 \\
\hline & $d\left(\mathrm{~kg} \mathrm{~kg}^{-1}\right)$ & 0,1451 & 0,1459 & 0,1479 & 0,1343 & 0,1548 \\
\hline \multirow[t]{4}{*}{4} & $\rho_{i}\left(\mathrm{~kg} \mathrm{~m}^{-3}\right)$ & 1385 & 1372 & 1373 & 1433 & 1369 \\
\hline & $\rho_{f}\left(\mathrm{~kg} \mathrm{~m}^{-3}\right)$ & 1627 & 1623 & 1622 & 1648 & 1627 \\
\hline & $a\left(\mathrm{~kg} \mathrm{~kg}^{-1}\right)$ & 25,98 & 27,89 & 28,711 & 22,03 & 25,97 \\
\hline & $d\left(\mathrm{~kg} \mathrm{~kg}^{-1}\right)$ & 0,1500 & 0,1485 & 0,1508 & 0,1262 & 0,1596 \\
\hline \multirow[t]{4}{*}{5} & $\rho_{i}\left(\mathrm{~kg} \mathrm{~m}^{-3}\right)$ & 1372 & 1341 & 1362 & 1430 & 1351 \\
\hline & $\rho_{f}\left(\mathrm{~kg} \mathrm{~m}^{-3}\right)$ & 1623 & 1620 & 1593 & 1581 & 1605 \\
\hline & $a\left(\mathrm{~kg} \mathrm{~kg}^{-1}\right)$ & 22,69 & 16,54 & 28,54 & 25,81 & 23,47 \\
\hline & $d\left(\mathrm{~kg} \mathrm{~kg}^{-1}\right)$ & 0,1446 & 0,1535 & 0,1472 & 0,1389 & 0,1602 \\
\hline
\end{tabular}


(a)
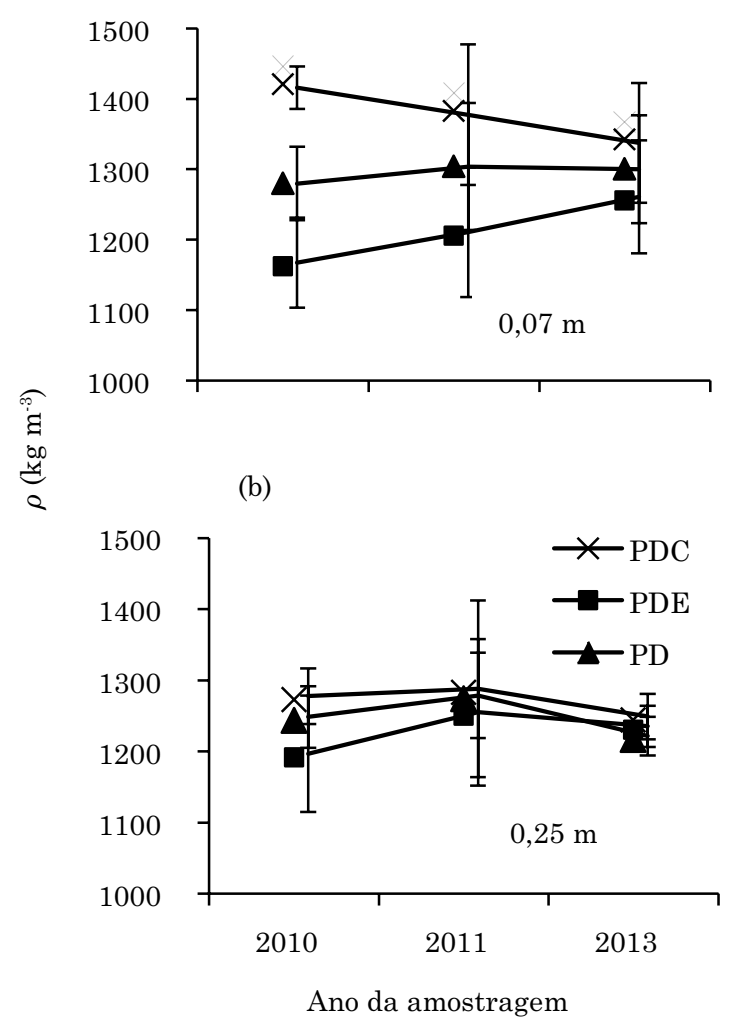

Figura 5. Densidade do solo ao longo do tempo após compactação (PDC), em 2010 (três tráfegos de trator) e 2011 (um tráfego de trator); e escarificação (PDE), em 2010, comparados ao solo em plantio direto (PD), nas profundidades de 0,07 e $0,25 \mathrm{~m}$. Barras verticais representam o desvio-padrão.

ao que parece, foi o resultado da interação ocorrida entre capacidade intrínseca do solo, frequência das diferentes cargas aplicadas pelas máquinas, condições de conteúdo de água do solo nas ocasiões de tráfego e atividade biológica. Esse caso particular não pode ser generalizado, mesmo para solos com grande resiliência, mas ele sugere que nesses solos operações de descompactação mecanizada podem ser desnecessárias.

Embora este trabalho trate da reversão da compactação, foi útil fazer um comparativo da recuperação da estrutura do solo após compactação com a reconsolidação do solo após revolvimento. Diversas evidências demonstram que a reconsolidação do solo após escarificação pode acontecer num período pouco maior que um ano (Hamilton-Manns et al., 2002; Álvarez et al., 2009). Para a camada superficial do Latossolo Vermelho distroférrico típico deste estudo, a diminuição da $\rho$ após compactação (PDC) foi tão rápida quanto o aumento da densidade após reconsolidação (PDE). Num período de dois anos, a $\rho$ em ambas as condições se aproximou da $\rho$ em plantio direto (PD) (Figura 5).

Conjuntamente, as análises em laboratório e em campo indicaram papel importante do processo de contração e expansão na reversão da compactação do Latossolo Vermelho distroférrico típico. Além da mudança na densidade observada neste trabalho, seria útil conhecer o potencial da contração e expansão para a manutenção de funções ecológicas do solo, como infiltração e aeração em diferentes solos sob plantio direto. Assim, uma análise mais abrangente pode subsidiar a decisão de se fazer ou não a intervenção para descompactação do solo.

\section{CONCLUSÕES}

O aumento da densidade do solo decorrente da contração por forças matriciais pode ser expressivo; neste estudo, isso se tornou relevante quando o conteúdo de água no solo foi menor que o do ponto de murcha permanente (1,5 $\mathrm{MPa})$.

Os repetidos ciclos de contração e expansão podem gradativamente diminuir a densidade do solo, reverter o processo de compactação e recuperar a estrutura de solos compactados. Sequências de contração e expansão no solo em campo podem diminuir a compactação superficial dele, que é comum no plantio direto.

\section{AGRADECIMENTO}

Ao Programa de Bolsas de Iniciação Científica ou Auxílio à Pesquisa - FIPE Júnior - Edital 012/2013 - PRPGP/UFSM.

\section{REFERÊNCIAS}

Álvarez CR, Duggan MT, Chamorro ER, D’Ambrosio D, Taboada MA. Descompactación de suelos franco limosos en siembra directa: Efectos sobre las propiedades edáficas y los cultivos. Ci Suelo. 2009;27:159-69.

Bastos RS, Mendonça ES, Alvarez V VH, Corrêa MT. Formação e estabilização de agregados do solo decorrentes da adição de compostos orgânicos com diferentes características hidrofóbicas. R Bras Ci Solo. 2005;29:11-20.

Bavoso MA, Silva AP, Figueiredo GC, Tormena CA, Giarola NFB. Resiliência física de dois Latossolos Vermelhos sob plantio direto. R Bras Ci Solo. 2012;36:1892-1904.

Bengough AG, McKenzie BM, Hallett PD, Valentine TA. Root elongation, water stress, and mechanical impedance: A review of limiting stresses and beneficial root tip traits. J Exp Bot. 2011;62:59-68.

Busscher WJ. Adjustment of flat-tipped penetrometer resistance data to a common water content. Trans ASAE. 1990;33:519-24. 
Chinn C, Pillai U. Self-repair of compacted Vertisols from Central Queensland, Australia. Geoderma. 2008;144:491-501.

Cooperativa dos Agricultores de Plantio Direto - Cooplantio. Plantio direto - Reduzir custos de produção, melhorar a produtividade e preservar os recursos naturais. 2012 [acesso 2012 dez 21]. Disponível em: http://www1.cooplantio.com.br/ quem-somos/a-cooplantio.aspx.

Decagon Devices. Operator's manual version 1.3 WP4 dewpointmeter. New York: Pullman; 2000.

Federação Brasileira de Plantio Direto na Palha - Febrapdp. Área de plantio direto no Brasil [acesso 2014 set 20]. Disponível em: http://www.febrapdp.org.br/download/Ev_area_pd_brasil.pdf.

Gebhardt S, Fleige H, Horn R. Anisotropic shrinkage of mineral and organic soils and its impact on soil hydraulic properties. Soil Till Res. 2012;125:96-104.

Genro Junior AS, Reinert DJ, Reichert JM. Variabilidade temporal da resistência à penetração de um Latossolo Argiloso sob semeadura direta com rotação de culturas. R Bras Ci Solo. 2004;28:477-84.

Gubiani PI, Reichert JM, Reinert DJ. Indicadores hídricomecânicos de compactação do solo e crescimento de plantas. $\mathrm{R}$ Bras Ci Solo. 2013a;37:1-10.

Gubiani PI, Goulart RZ, Reichert JM, Reinert DJ. Crescimento e produção de milho associados com o intervalo hídrico ótimo. $\mathrm{R}$ Bras Ci Solo. 2013b;37:1502-11.

Gubiani PI. Regularidade de resposta da cultura do milho à compactação do solo [tese]. Santa Maria: Universidade Federal de Santa Maria; 2012.

Hamilton-Manns M, Ross CW, Horne DJ, Baker CJ. Subsoil loosening does little to enhance the transition to no-tillage on a structurally degraded soil. Soil Till Res. 2002;68:109-19.

Horn R; Smucker A. Structure formation and its consequences for gas and water transport in unsaturated arable and forest soils. Soil Till Res. 2005;82:5-14.

Kämpf N, Curi N. Argilominerais em solos brasileiros. In: Curi N, Marques JJ, Guilherme LRG, Lima JM, Lopes AS, Alvarez V VH, editores. Tópicos em ciência do solo. Viçosa, MG: Sociedade Brasileira de Ciência do Solo; 2003. v.3, p.1-54.
Klein VA. Física do solo. 3a ed. Passo Fundo: Universidade de Passo Fundo; 2014.

Klein VA, Baseggio M, Madalosso T. Indicadores da qualidade física de um Latossolo Vermelho distrófico típico sob plantio direto escarificado. Ci Rural. 2009;39:2475-81.

Pires LF, Bacchi OOS. Mudanças na estrutura do solo avaliada com uso de tomografia computadorizada. Pesq Agropec Bras. 2010;45:391-400.

Peng X, Horn H, Petha S, Smucker A. Quantification of soil shrinkage in $2 \mathrm{D}$ by digital image processing of soil surface. Soil Till Res. 2006;91:173-80.

Reichert JM, Suzuki LEAS, Reinert DJ, Horn R, Hakansson I. Reference bulk density and critical degree-of-compactness for no-till crop production in subtropical highly weathered soils. Soil Till Res. 2009;102:242-54.

Reichert JM, Suzuki LEAS, Reinert DJ. Compactação do solo em sistemas agropecuários e florestais: Identificação, efeitos, limites críticos e mitigação. In: Ceretta CA, Silva LS, Reichert JM, editores. Tópicos em ciência do solo. Viçosa, MG: Sociedade Brasileira de Ciência do Solo; 2007. v.5, p.49-134.

Statistical Analysis System - SAS. SAS/STAT procedure guide for personal computers. 5a ed. Cary: Statistical Analysis System Institute; 1999

Secco D, Reinert DJ, Reichert JM, Silva VR. Atributos físicos e rendimento de grãos de trigo, soja e milho em dois Latossolos compactados e escarificados. Ci Rural. 2009;39:58-64.

Silva AP, Kay BD, Perfect E. Characterization of the least limiting water range of soils. Soil Sci Soc Am J. 1994;58:1775-81.

Suzuki LEAS, Reinert DJ, Reichert JM, Lima CLR. Estimativa da susceptibilidade à compactação e do suporte de carga do solo com base em propriedades físicas de solos do Rio Grande do Sul. R Bras Ci Solo. 2008;32:963-73.

Viana JHM, Fernandes Filho EI, Schaefer CEGR. Efeitos de ciclos de umedecimento e secagem na reorganização da estrutura microgranular de Latossolos. R Bras Ci Solo. 2004;28:11-9. 